Sharif University of Technology
Scientia Iranica
Transactions E: Industrial Engineering
hCIENTIA

\title{
Robust-fuzzy model for supplier selection under uncertainty: An application to the automobile industry
}

\author{
M. Rabieh ${ }^{\mathrm{a}, *}$, M. Modarres ${ }^{\mathrm{b}}$, and A. Azar \\ a. Department of Industrial Management, Shahid Beheshti University, Tehran, Iran. \\ b. Department of Industrial Engineering, Sharif University of Technology, Tehran, Iran. \\ c. Department of Industrial Management, Tarbiat Modares University, Tehran, Iran.
}

Received 3 March 2016; received in revised form 22 February 2017; accepted 30 May 2017

\section{KEYWORDS}

Supplier selection; Uncertainty;

Robust optimization;

Fuzzy programming;

Robust-fuzzy model;

Auto industry.

\begin{abstract}
This paper proposes an innovative robust-fuzzy method for multi-objective, multi-period supplier selection problem under multiple uncertainties. This approach integrates robust optimization and fuzzy programming. Uncertain parameters are modeled as random variables that take value within a symmetrical interval. However, due to the complexity or ambiguity of some real world problems and especially the nature of some of the available input data, the length of interval is also highly uncertain. This ambiguity motivated us to present a new approach, which could be applicable to multiple uncertainties conditions. Thus, in our approach, the half-length of these intervals is also represented by fuzzy membership function. We develop a model and a solution approach to select suppliers by considering risk. The proposed method is applied to a real case of supplier selection in automobile industry under uncertainty and ambiguity conditions. To verify the proposed model, we evaluate the results by simulation technique and compare values of objective function under different scenarios.
\end{abstract}

(C) 2018 Sharif University of Technology. All rights reserved.

\section{Introduction}

Supplier selection is an important strategic decision making for managers in supply chain management [1]. One can find numerous studies and models in the supplier selection literature, see Section 2. The decision making models in the literature are basically developed to answer the following questions: How many suppliers are needed? Which suppliers should be selected? What is the optimal ordering policy to each supplier? One can find many deterministic models developed in the literature to answer these questions.

The main disadvantage of deterministic models is

\footnotetext{
*. Corresponding author.

E-mail address: m_rabieh@sbu.ac.ir (M. Rabieh)
}

doi: $10.24200 /$ sci. 2017.4456 that they fail to be accountable when encountering the uncertain nature of real world systems.

On the other hand, risk and uncertainty management is an inherent part of supplier selection. The importance and the effects of uncertainty in supplier selection have been widely emphasized in the supply chain literature [1-3].

To handle the effect of uncertainty of input parameters, either probabilistic or fuzzy approaches can be applied. However, in many real cases, due to the lack of enough historical data to fit appropriate probability distribution for uncertain parameters, stochastic programming cannot be applied. Furthermore, computational complexity of stochastic programming models is another limitation on their application. Therefore, robust optimization methods can be considered as an efficient alternative for handling this type of uncertainty [3]. Recently, some researchers have 
developed the robust optimization approach to handle uncertainties in supplier selection $[1,2]$. Moreover, robust optimization has emerged as a methodology for managing supply chain risks. Robustness plays an important role in sustainability of supply with uncertain input data. Robustness of supply chain has been the frontier of supply chain research [4].

Fuzzy programming is another method for handling a type of uncertain parameters, which are usually expressed in linguistic and vague terms and then transformed into fuzzy numbers.

In real cases, due to the vagueness of the information about parameters, deterministic methods are not suitable for effective supplier selection. Under these conditions, the theory of fuzzy sets is one of the best tools to handle the uncertainty [5]. Fuzzy programming based on fuzzy sets theory is utilized when the information for supplier selection is vague and imprecise.

In this research, we develop a new method of robust optimization to handle uncertainties. In robust optimization, the models are developed based on the assumption that no knowledge about the distribution of uncertain parameters exists. In these models, uncertainty of parameters is modeled as symmetric and bounded random variables. Every symmetrical interval is characterized by two numbers: middle number of interval (nominal value) and half-length of interval. It is assumed that these numbers are given. However, in real world applications, forecasting their exact values seems to be difficult or even impossible. In fact, errors and ambiguity in forecasting and measuring model parameters make the decision making model prone to many resources underutilization and/or overutilization problems. In our real case study, we faced the problem of determining the half-length of interval of supplier's capacity parameter. That case motivated us to provide an innovative robust-fuzzy model. In this approach, the half-length of interval is presented by a fuzzy number. In fact, the main innovation of this research is combining the robustness and fuzziness concepts in the presence of multiple uncertainties.

In order to illustrate our proposed Robust-Fuzzy (R-F) method, it is implemented for a real case of supplier selection in automobile industry under multiple uncertainties. Under various conditions, the results proved to be accurate enough in comparison with the results by simulation techniques. In other words, the proposed model can properly manage a variety of uncertain conditions and provide useful and reliable information for flexible decision making processes.

The rest of this paper is organized as follows. In Section 2, literature review is presented. In Section 3, the case study is described. Steps of implementing the model case are presented in Section 4. Section 5 presents the methodology of robust-fuzzy (R-
F) method. In Section 6, the mathematical model is formulated. Data collection and parameters are described in Section 7. Computational result is presented in Section 8 and, finally, some concluding remarks are given in Section 9.

\section{Literature review}

Due to the importance of supplier selection decision, an extensive literature review of both deterministic and uncertain models is presented to address this type of decision. In the section pertinent to uncertain models, three categories of the mentioned models are stated in stochastic, fuzzy, and robust forms. Moreover, relevant researches are reviewed for each category. Finally, considering the strengths and weaknesses of each category and the current gap in the literature, the contribution of the article is presented.

\subsection{Deterministic models}

In this section, a brief overview of relevant studies of deterministic supplier selection models is presented. Benton [6] used Lagrange relaxation to develop a non-linear program for supplier selection under various conditions including multiple suppliers, multiple items, resource limitations, and quantity discount. Ghodsypour and O'Brien [7] used integrated Analytical Hierarchy Process (AHP) with mixed integer programming to develop a Decision Support System (DSS) in order to reduce the number of suppliers. Ghodsypour and O'Brien [8] also developed a model by integration of AHP and linear programming model to take into account both qualitative and quantitative factors. In a further development, Ghodsypour and O'Brien [9] presented a mixed integer non-linear programming model by considering the total cost of logistics for multiple sourcing cases. Basnet and Leang [10] investigated the problem of supplier selection by considering the lot-sizing. Ustun and AkhtarDemirtas [11] used an integrated approach of Analytic Network Process (ANP) and Multi-Objective Mixed Integer Linear Programming (MOMILP) for supplier selection problem. Their approach considered both tangible and intangible factors in choosing the best suppliers and defined the optimum quantities among selected suppliers to maximize the Total Value of Purchasing (TVP), minimize the total cost and total defect rate, and balance the total cost among periods. Finally, Mendoza and Ventura [12] presented two mixed integer nonlinear programming models to select the best suppliers and determine order quantities, while minimizing the annual ordering, inventory holding, and purchasing costs with respect to suppliers' capacity and quality constraints. Their research integrated the issues of inventory management and supplier selection.

The literature on deterministic models considers the following as significant items: 
- Some of these models are multi-objective (see [10]);

- Some of these models consider some important characteristics such as multiple suppliers and multiple items, simultaneously (see [12]);

- Obviously, these models are not applicable to uncertain environments.

\subsection{Uncertain models}

In this section, a brief overview of relevant studies of uncertain supplier selection models (i.e., fuzzy, stochastic, and robust models) is presented.

\subsubsection{Fuzzy models}

Some researchers developed fuzzy models. Kumar et al. [13] used a fuzzy mixed integer goal programming approach to solve the vendor selection problem in case of multiple objectives. Chen et al. [14] presented a fuzzy decision making approach to solve the supplier selection problem. They used linguistic values to evaluate the ratings for a number of quantitative and qualitative factors including quality, price, flexibility, and delivery performance. Their model was shown to be a good tool for supplier selection decision making situation. Kilic [15] presented a new integrated approach, including fuzzy TOPSIS method and a mixed integer linear programming model, to select the suitable supplier in a multi-item/multi-supplier environment. In the first stage of this approach, importance value of each supplier for each product was obtained via fuzzy TOPSIS. In the second stage, outputs of the first stage were used as inputs to the proposed mathematical model; the best suppliers were selected; and the amount of order for each product (item) to each supplier was determined via solving the model. Nazari-Shirkohi et al. [16] proposed an interactive Fuzzy Multi-Objective Linear Programming (FMOLP) model to solve the multi-objective supplier selection problem and order allocation under multiple price levels and multiple products. The model attempted to minimize cost as well as numbers of defective units and late delivered units ordered from suppliers. Finally, Roshandel et al. [17] presented a fuzzy hierarchical TOPSIS approach to select and evaluate suppliers of imported raw materials for the detergent production industry in Iran. The authors claimed that their approach overcame disadvantages of a prior well-known method in the area of fuzzy TOPSIS (Chen's method).

Soufi Neyestani et al. [18] presented an evolutionary algorithm for supplier order allocation with fuzzy parameters considering linear volume discount. As some information, such as buyer demand and supplier delivery rate, was uncertain, the authors applied fuzzy sets to handle the uncertainty.

Mahmoudi et al. [19] extended fuzzy VIKOR using an efficient fuzzy distance measure to solve applicable Supplier Selection Problem (SSP) under group decision-making. The authors claimed that, as SSP parameters were often imprecise, vague, uncertain, or incomplete in real-life situations, fuzzy sets theory was the best developed approach to handle the uncertainties.

There are some general shortcomings in fuzzy approaches:

- Fuzzy approaches are only applicable in the condition of uncertainty in a fuzzy form;

- Determining the real membership function of the fuzzy numbers is not easy;

- In all the above-mentioned studies, the issues of single uncertainty are investigated, but not fully matched with real world cases.

\subsubsection{Stochastic models}

Kasilingam and Lee [20] developed a chanceconstrained integer programming formulation for vendor selection and order allocation by minimizing costs. Their model also considered the lead time requirements and vendors capacities, and used a chance constraint due to the stochastic nature of demand.

Feng et al. [21] presented a Stochastic Integer Programming (SIP) method for simultaneous selection of tolerances and suppliers based on the quality loss function and process capability indices. In this research, to achieve the required quality level and minimum total cost, process capability indices and quality loss function were also incorporated in a mathematical model.

Bonser and $\mathrm{Wu}[22]$ developed a stochastic program for solving procurement problem in an electrical utilities firm under uncertain demand and market price.

Several other works have also considered this type of uncertainty in supplier selection problem. Seshadri et al. [23] developed a probabilistic bidding model to represent the connections in multiple-sourcing and their consequences. Their bidding model investigated the effect of multiple-sourcing on competitive behavior prior to supplier selection. Ranjbar Tezenji et al. [24] presented an integrated model for supplier location-selection and order allocation under capacity constraints in an uncertain environment. The authors considered the costs to be uncertain and stochastic.

In many real cases, applications of stochastic programming are limited, because:

- Enough historical data about appropriate probability distribution for uncertain parameters is not usually available;

- These models have some computational complexities [3].

The aforementioned shortcomings motivated us to select an appropriate alternative method, especially for our real case with especial characteristics. In such 
cases, robust optimization methods could be adopted, because these methods (especially Bertsimas and Sim's approach) are more practical.

In fact, robust optimization methods try to present solutions which are less sensitive to changes of parameters [3].

\subsubsection{Robust models}

Recently, some researchers have developed the robust optimization approach to handle uncertainties in supplier selection $[1,2]$.

Li and Zabinsky [1] developed stochastic mathematical programming models to capture the risk associated with uncertain customer demand and supplier capacity. They used multi-parametric programming techniques to analyze tradeoffs and determine a robust set of suppliers with balanced costs and risks. In fact, the final result of their research was robust selection of suppliers.

Nam et al. [2] developed a model for robust supply base management under both demand and supply uncertainties. In fact, in supply base management under the condition of uncertainty, having an understanding of benefits associated with a contractor that has an optimal number of suppliers is important. Briefly, the model determined the optimal robust number of suppliers that a contractor should maintain in its supply base in the condition of uncertainty.

The literature on the robust models shows that:

- Only the issue of single uncertainty has been investigated;

- There has been no attention to robust optimization methods, especially Bertsimas and Sim's approach as an efficient method.

Considering shortcomings of each section (deterministic and uncertain models), the literature has paid little attention to the following items:

- Simultaneous attention to characteristics such as multiple items, multiple suppliers, multiple objectives (goals), multiple periods, and multiple products;

- Robust optimization methods, especially Bertsimas and Sim's approach as an efficient method;

- Multiple uncertainties.

This research aims to fill these gaps. For example, the proposed comprehensive model incorporates assumptions and characteristics such as multiple items (parts), multiple suppliers, multiple objectives (goals), multiple periods, multiple products (automobiles), multiple uncertainties, and two-echelon supply chain including suppliers and manufactures (plants). Also, in this research, the important criteria of price, quality, and delivery are considered.
The contribution of this research regarding the value it adds to the theory is presented as follows.

In fact, the main contribution of this research is combining the robustness and fuzziness concepts in the presence of multiple uncertainties. In other words, this research proposes a novel approach for multiple uncertainties.

\section{Case application}

By generating high employment rate, high financial turnover, and significant rate of deals with foreign automotive industry, the automotive industry is one of the important industries after oil and gas industry. This industry significantly affects the national economy. Therefore, its supply chain is one of the most dynamic chains in industries. This chain provides thousands of parts for each vehicle.

While some parts are supplied by a single source, many others have multiple sources, which include more than 70 percent of the total value for each vehicle. Considering the number of suppliers, which can be from one to four, we implement the proposed supplier selection method for Iran Khodro, the largest automotive company in Iran.

Due to the high volume of required parts, choosing suitable suppliers is of great importance for this industry.

Some existing data (supplier's capacity and transportation cost) are uncertain by their nature. Thus, due to the existence of uncertain data, we should adopt robust optimization for supplier selection planning. This motivated us to develop a new method named robust-fuzzy method.

Although the problem is formulated in its general form, we collected data for two models of vehicles, namely, Peugeot 405 and Pars, only for Tehran plant, which is the largest and most important plant of the company.

\section{Methodology of robust-fuzzy}

As mentioned, one way of dealing with uncertainty is by stochastic programming modeling. The main shortcomings of stochastic programming approach are: (a) lack of existence of distribution function of data, and (b) computational challenges [25]. The concept and advantages of robust approach, in general, and supply chain planning, in particular, are illustrated in Figure 1. Deterministic approaches obtain the solution based on averaging or "good guess". In contrast, robust approaches provide a solution which is "nearoptimal". Although the resulting cost is more than that of deterministic approach, the solution is more reliable. In other words, taking into account the variability of 


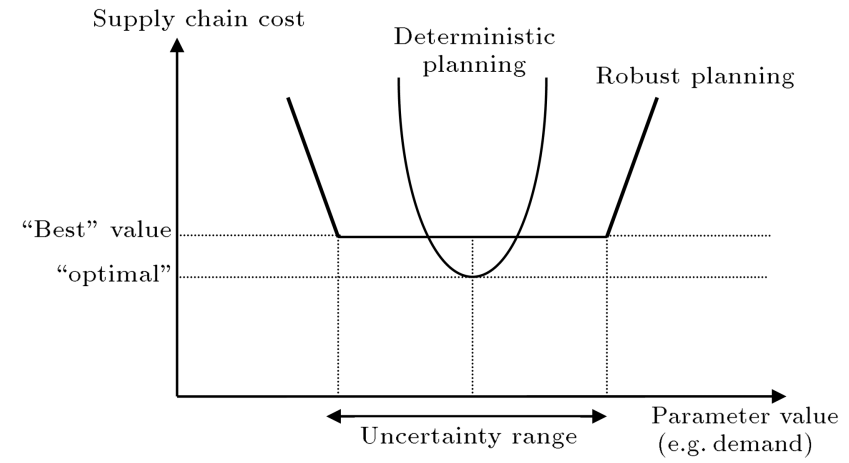

Figure 1. The effect of robust planning on total supply chain cost [25].

parameters in a range of values, the solution is still reliable with high confidence [26].

Soyster [27] took the very first step toward using robust optimization, proposing linear programming model. This model produced solutions that were too conservative. The next steps were by Ben-Tal and Nemirovski [28-30], El-Ghaoui et al. [31], and ElGhaoui and Lebert [32] to address over-conservatism. They applied robust optimization to linear programming problem with ellipsoidal uncertainty sets, thus obtaining conic quadratic programs.

Bertsimas and Sim [33] presented a new approach specifically tailored for polyhedral uncertainty, which led to linear robust counterparts, i.e., it retained the advantages of linear framework of Soyster (1973) [27] while controlling conservativeness level of the solution. Thus, their method was also appropriate for solving discrete optimization problems, and that is the reason we have adopted Bertsimas and Sim's model.

As explained before, in robust optimization approach, uncertain data take value within an interval. Although no distribution function for a random variable is available, the length of interval is given. However, in some real cases, it is difficult for the decision maker to determine the exact interval length (or half-length if it is symmetrical). If the decision makers consider a high half-length of interval, then the level of conservatism and, consequently, the cost would be increased. On the other hand, if the interval halflength is considered low, the risk would be increased. Furthermore, in some situations, the decision maker suggests ambiguous interval half-length. To overcome this problem, we develop an innovative approach and represent the interval half-length in terms of fuzzy number. In fact, we develop a Robust-Fuzzy (R-F) method, which integrates the concepts and techniques of robust optimization (Bertsimas and Sim's approach) and fuzzy programming (Werners' approach) [34,35].

In addition to having the applicable features of Bertsimias and Sim's model, this method is properly responsive to the ambiguity of the interval half-length for the decision maker and provides necessary flexibility for decision makers. In other words, this method is able to solve the problems in multiple uncertainty conditions. follows:

Consider nominal mixed integer programming, as

$$
\begin{aligned}
& \text { Min } \quad c^{\prime} x, \\
& \text { S.t. } \quad A x \leq b, \quad l \leq x \leq u, \\
& x_{i} \in Z, i=1, \ldots, k
\end{aligned}
$$

Without loss of generality, we assume that only the elements of matrices $A$ and $c^{\prime}$ are uncertain. In practice, the mean values of coefficients $a_{i j}$ and its interval half-length, $\hat{a}_{i j}$, as well as $c_{j}$ and $d_{j}$ are estimated. The model of data uncertainty is represented in the following [33]:

(a) Uncertainty for matrix $A$ : let $N=\{1,2, . ., n\}$. Each entry $a_{i j}, j \in N$, is modeled as an independent, symmetric, and bounded random variable (with unknown distribution) $\tilde{a_{i j}}, j \in N$, that takes values in $\left[a_{i j}-\hat{a}_{i j}, a_{i j}+\hat{a}_{i j}\right]$;

(b) Uncertainty for cost vector $c$ : Each entry $c_{j}, j \in$ $N$, takes values in $\left[c_{j}, c_{j}+d_{j}\right]$, where $d_{j}$ represents the deviation from the nominal cost coefficient, $c_{j}$.

Furthermore, we define that $\left|J_{i}\right|$ represents the number of uncertain components in the $i$ th constraint; and $\Gamma_{i}, i=0,1, \ldots, m$, takes values in $[0,|J|]$.

The role of parameter $\Gamma_{i}$ in constraint $i$ is to adjust the robustness of the proposed method to the level of conservatism of the solution. Similarly, the level of robustness in objective function is controlled by parameter $\Gamma_{0}$. Therefore, the robust counterpart of Problem (1) is as follows:

$$
\begin{aligned}
& \text { Min } z=c^{\prime} x+z_{0} \Gamma_{0}+\sum_{j \in j_{0}} p_{0 j}, \\
& \text { S.t. }: \sum_{j} a_{i j} x_{j}+z_{i} \Gamma_{i}+\sum_{j \in j_{i}} p_{i j} \leq b_{i}, \\
& z_{0}+p_{0 j} \geq d_{j} y_{j}, \quad z_{i}+p_{i j} \geq \hat{a}_{i j} y_{j}, \\
& p_{i j} \geq 0, \quad y_{i j} \geq 0, \quad z_{i} \geq 0, \\
& -y_{j} \leq x_{j} \leq y_{j} \forall j \quad l_{j} \leq x_{j} \leq u_{j} \forall j \quad x_{i} \in z .
\end{aligned}
$$

Model 2 is Bertsimas and Sim's robust counterpart.

\subsection{Linear programming problem with fuzzy resources: Werners' approach}

Consider the following general fuzzy linear programming model with fuzzy resources (nonsymmetrical 
model):

$\operatorname{Max} Z=f(x)=C X$,

S.t. : $(A X)_{i} \tilde{\leq} b_{i}, \quad i=1,2, \ldots, m$, $X \geq 0$

or

$\operatorname{Max} Z=f(x)=C X$,

S.t. : $(A x)_{i} \leq \tilde{b}_{i}, \quad i=1,2, \ldots, m$,

$X \geq 0$,

where $\tilde{b}_{i}$, and $\forall i$ is in $\left[b_{i}, b_{i}+p_{i}\right]$ with given $p_{i}$.

To make this model a symmetrical one, Werners $[34,35]$ proposed that its objective function should be of fuzzy type. Therefore, fuzzy resource's tolerance $p_{i}$ is assumed to be given and certain. He initially offered the following definitions for inf $f$ and $\sup f$ :

$$
\begin{aligned}
& Z^{0}=\inf f=\operatorname{Max} C X, \\
& \text { S.t. }:(A x)_{i} \leq b_{i}, \\
& X \geq 0, \\
& Z^{1}=\sup f=\operatorname{Max} C X, \\
& \text { S.t. }:(A x)_{i} \leq b_{i}+p_{i}, \\
& X \geq 0 .
\end{aligned}
$$

Membership function for objective function and constraints is shown in Figures 2 and 3.

To achieve optimal solutions, we use Max-Min operator. Therefore, fuzzy linear programming can be converted to a symmetrical model as follows:

$\operatorname{Max}_{x \geq 0} \lambda$, where $\lambda=\operatorname{Min}\left[\mu_{0}(x), \mu_{1}(x), \ldots \mu_{m}(x)\right]$.

$$
\mu_{0}(x)= \begin{cases}0 & \text { if } C X \leq Z^{0} \\ \frac{C X-Z^{0}}{Z^{1}-Z^{0}} & \text { if } \quad Z^{0} \leq C X \leq Z^{1} \\ 1 & \text { if } \quad Z^{1} \leq C X\end{cases}
$$

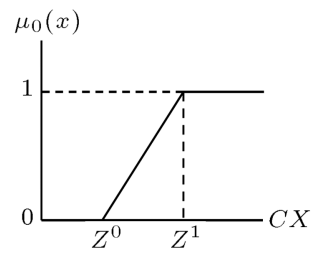

Figure 2. Membership function of the objective function.

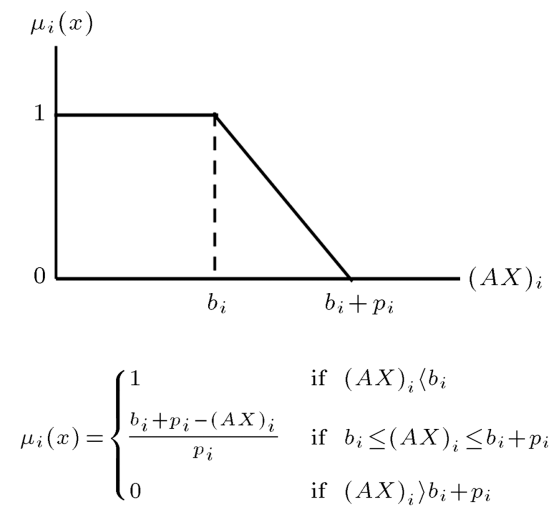

Figure 3. Membership function of a constraint.

\subsection{Innovative robust-fuzzy approach}

As explained before, our robust-fuzzy approach integrates techniques of robust optimization (Bertsimas and Sim's approach) and fuzzy programming (Werners' approach).

In this section, we summarize the model by considering the uncertainty of decision makers in determining interval half-length of the parameter as a fuzzy number, $\tilde{\hat{b}}_{i}$, with the membership function shown in Figure 4.

The resulting model is a linear programming one with fuzzy resources (nonsymmetrical model), which can be converted into a symmetric one and, finally, into the following deterministic model:

$$
\begin{aligned}
& \operatorname{Max} z=\lambda \text {; } \\
& c^{\prime} x+z_{0} \Gamma_{0}+\sum_{j \in j_{0}} p_{0 j}+\left(Z^{1}-Z^{0}\right) \lambda \leq Z^{1}, \\
& \text { S.t. : } \sum_{j} a_{i j} x_{j}+z_{i} \Gamma_{i}+\sum_{j \in j_{i}} p_{i j}+q_{i} \leq b_{i} \quad \forall i \text {, } \\
& z_{0}+p_{0 j} \geq d_{j} y_{j} \quad \forall j \in J_{0}, \\
& z_{i}+p_{i j} \geq \hat{a}_{i j} y_{j} \quad \forall i \neq 0, j \in J_{i}, \\
& z_{i}+p_{i 0}-\lambda\left(\hat{b}_{i}-\hat{b}_{i_{\min }}\right) \geq \hat{b}_{i_{\min }} \quad \forall i, j \in J_{i}, \\
& p_{i j} \geq 0 \quad \forall i, j, \\
& y_{i j} \geq 0 \quad \forall i, j, \\
& z_{i} \geq 0 \quad \forall i \\
& -y_{j} \leq x_{j} \leq y_{j} \quad \forall j \\
& l_{j} \leq x_{j} \leq u_{j} \quad \forall j, \\
& x_{i} \in z \quad i=1, \ldots, k \text {. }
\end{aligned}
$$

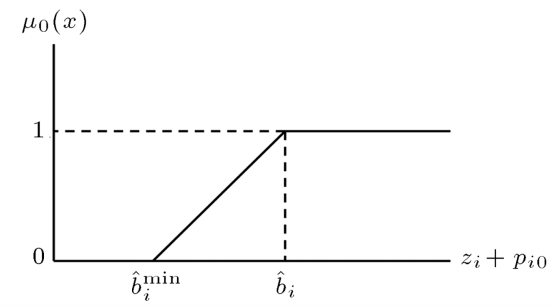

$$
\mu_{0}(x)= \begin{cases}0 & \text { if } z_{i}+p_{i 0} \leq \hat{b}_{i}^{\text {min }} \\ \frac{z_{i}+p_{i 0}-\hat{b}_{i}^{\text {min }}}{\hat{b}_{i}^{\text {med }}-\hat{b}_{i}^{\text {min }}} & \text { if } \hat{b}_{i}^{\text {min }} \leq z_{i}+p_{i 0} \leq \hat{b}_{i} \\ 1 & \text { if } \hat{b}_{i} \leq z_{i}+p_{i 0}\end{cases}
$$

Figure 4. Membership function of $\hat{b}_{i}$. 
In Model $6, P_{i j}$ and $q_{i}$ are robustness variables. When converting a nominal model to its robust counterpart model, robustness variables are added to the model.

The proposed method is more flexible than the base model (Bertsimas and Sim's model); also, it eliminates the decision maker's uncertainty about halflength intervals. In other words, the decision maker is able to reach pessimistic, optimistic, and more balanced results for them. If $\lambda=1$ (upper limit of the interval length), pessimistic results are obtained by Model 3; and if $\lambda=0$ (lower limit of the interval length), optimistic results are obtained.

In Section 8.1, Monte Carlo simulation experiments are conducted to justify robustness of solutions and estimate real level of conservatism against determinate budget. In fact, by simulation, the probability of constraints violation or risk rate will be determined. Briefly, the Monte Carlo simulation model is implemented to test and validate the robustness of the solution obtained via the robust model.

\section{Steps of our proposed approach}

Figure 5 shows the steps of this approach.

Data has been collected via intensive interviews with managers and experts as well as from the existing documents of Iran Khodro Company, and SAPCO Company, its main subsidiary, which is responsible for managing its supplying parts and components.

Also, profile of experts includes: five people with at least 10 years of experience and expertise, including the logistics manager, logistics project team leader,

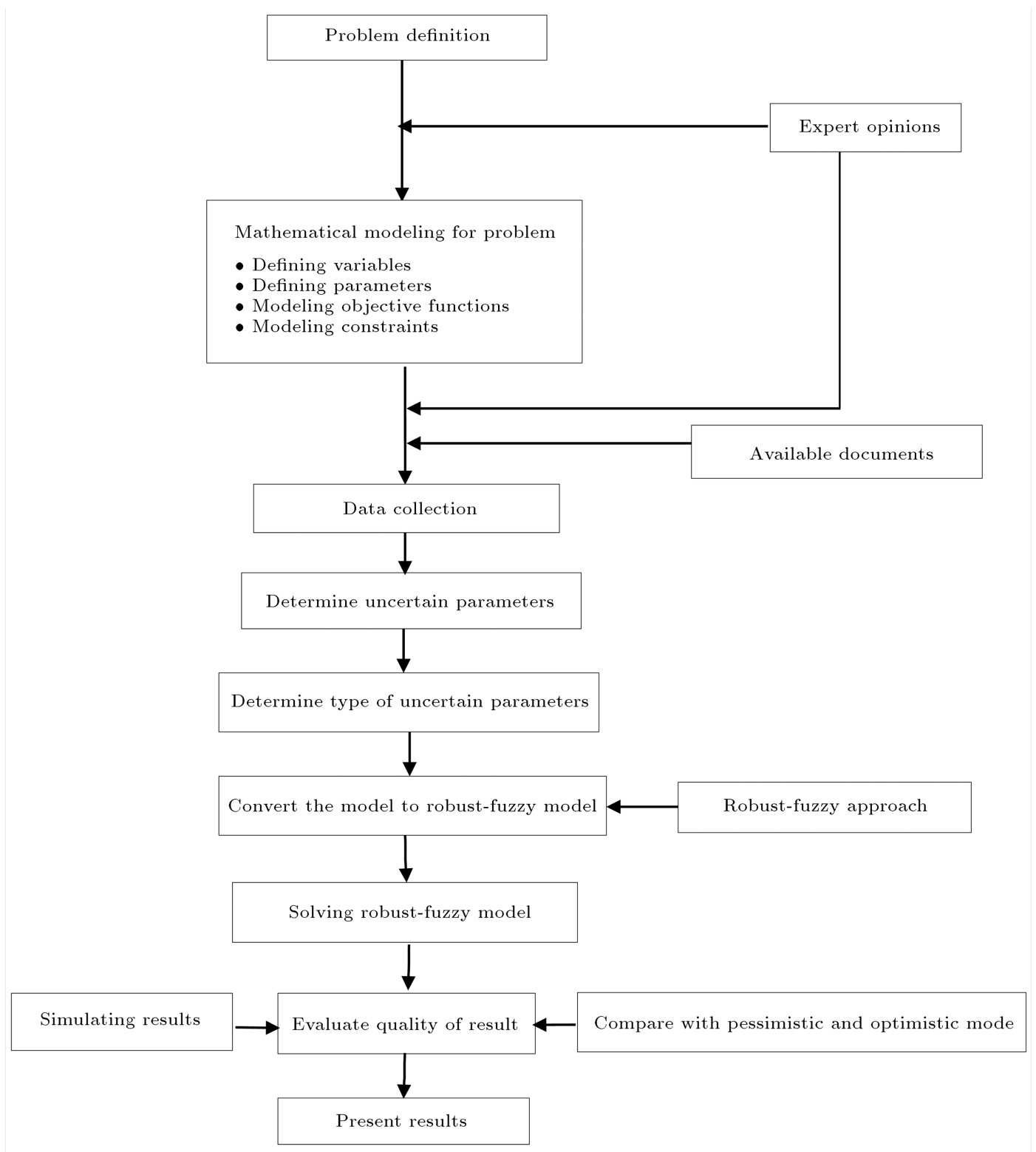

Figure 5. Steps of implementing the model case. 
head of logistics engineering, logistics engineering expert, and planning expert.

\section{Formulating the model}

In this section, we present the mathematical model of supplier selection problem.

\subsection{Notation}

\section{Sets}

I Set of products (automobiles)

$J \quad$ Set of plants

$M \quad$ Set of parts

$N \quad$ Set of suppliers

$T \quad$ Set of planning periods

$R \quad$ Set of objective functions (goals)

Certain parameters

$P_{i j t} \quad$ Production level of product $i$ in period $t$ for plant $j$

$C S_{m n} \quad$ Purchase cost of part $m$ from supplier $n$ $C_{m n} \quad$ Capacity of supplier $n$ to supply part $m$

$C h_{m n j} \quad$ Inventory cost in manufacturing plant j

$P P M_{m n} \quad$ Index of returned part $m$ to supplier $n$

$V C_{i m} \quad$ Consumption coefficient of part $m$ for product $i$

$D P_{m n} \quad$ On-time delivery number of part $m$ by supplier $n$

$W_{r} \quad$ Importance weight of objective $r$

$G_{r} \quad$ Value of goal $r$

$L T_{m} \quad$ Lead time of part $m$

$\alpha \quad$ Safety factor for determining permissible levels of inventory

$\beta \quad$ Factor for determining the minimum procurement from each supplier

$\Gamma \quad$ Robust protection level

Uncertain parameters

$C t_{m n j} \quad$ Transportation cost of part $m$ from supplier $n$ to plant $j$

$C_{m n} \quad$ Capacity of supplier $n$ for supplying part $m$

Issues related to uncertain parameters

$\bar{C} t_{m n j} \quad$ Nominal value of transportation cost of part $m$ from supplier $n$ to plant $j$

$\bar{C}_{m n} \quad$ Nominal value of capacity of supplier $n$ to supply part $m$

$\hat{C} t_{m n j} \quad$ Half-length of transportation cost of part $m$ from supplier $n$ to plant $j$

$\tilde{\hat{C}}_{m n} \quad$ Half-length of capacity of supplier $n$ to supply part $m$.
Decision variables

$S_{m n j t} \quad$ Supply rate of part $m$ from supplier $n$ in period $t$ for plant $j$

$I S_{m n j t} \quad$ Inventory level of part $m$ from supplier $n$ in period $t$ for plant $j$

$d_{i}^{+}, d_{i}^{-} \quad$ Deviation from goal $i$ (positive, negative)

$\lambda \quad$ Degree of constraint's satisfaction

\subsection{Mathematical model}

\subsubsection{Objective functions}

We consider three objectives for the model as follows:

- Minimizing the cost of supplying parts;

- Minimizing the defective parts supplied by suppliers;

- Maximizing on-time delivery.

The first objective function consists of purchasing cost, transportation cost from supplier to manufacturing plant, and holding cost. The second objective function is related to PPM (part per million) factor. This factor is one of the most important indices in this industry. In fact, it calculates the ratio of number of defects to the volume of shipment. It is clear that any suppliers that have lower PPM will attract more orders. The third objective is an important indicator, highly emphasized by industry experts as well as in the literature. Obviously, any suppliers that have more ontime delivery will attract more orders.

To consider all three objectives, we formulate the problem by applying goal programming technique.

\subsubsection{Constraints}

Generally, the constraints can be classified into three categories of functional constraints, goal constraints, and robust constraints.

Functional (main) constraints of the model are:

- Parts demand: Demand in each period depends on three factors: production level per product, consumption coefficient of part per product, and inventory.

- Inventory level, maximum and minimum: Inventory maximum and minimum levels depend on four factors: production level per product, consumption coefficient of part per product, lead time of part, and safety factor of determining permissible levels of inventory;

- Suppliers' capacity;

- The minimum quantity of a part which can be purchased from each supplier.

Due to the company's policy for purchasing from all suppliers of a part, the minimum quantity of a part which can be purchased from each supplier 
is constrained by a factor that determines the minimum procurement level from each supplier $(\beta)$.

\subsection{Nominal goal model}

The nominal goal model is:

$\operatorname{Min} Z=\sum_{r=1}^{r} w_{r}\left(d_{r}^{+}, d_{r}^{-}\right)=\frac{w_{1} d_{1}^{+}}{G 1}+\frac{w_{2} d_{2}^{+}}{G 2}+\frac{w_{3} d_{3}^{-}}{G 3}$,

S.t. :

$$
\begin{aligned}
\sum_{m=1}^{M} & \sum_{n=1}^{N} \sum_{j=1}^{J} \sum_{t=1}^{T} C S_{m n} S_{m n t j} \\
& +\sum_{m=1}^{M} \sum_{n=1}^{N} \sum_{j=1}^{J} \sum_{t=1}^{T} C t_{m n j} S_{m n t j} \\
& +\sum_{m=1}^{M} \sum_{n=1}^{N} \sum_{j=1}^{J} \sum_{t=1}^{T} C h_{m n} I S_{m n t j}+d_{1}^{-}-d_{1}^{+}=G_{1},
\end{aligned}
$$$$
\sum_{i=1}^{I} \sum_{m=1}^{M} \sum_{n=1}^{N} \sum_{t=1}^{T} P P M_{m n} S_{m n t}+d_{2}^{-}-d_{2}^{+}=G_{2}
$$$$
\sum_{i=1}^{I} \sum_{m=1}^{M} \sum_{n=1}^{N} \sum_{t=1}^{T} D P_{m n} S_{m n t}+d_{3}^{-}-d_{3}^{+}=G_{3}
$$$$
\sum_{n=1}^{n} S_{m n t j}=\sum_{i=1}^{i} V C_{i m} P_{i j t}-\sum_{n=1}^{n} I S_{m n j t-1}
$$$$
+\sum_{n=1}^{i} I S_{m n j t} \quad \forall j, m, t,
$$$$
\sum_{n=1}^{n} I S_{m n j t} \geq \sum_{i=1}^{i} V C_{i m} \times P_{i j t} / 30 \times \alpha \times L T_{m}
$$

$\forall j, m, t$,

$$
\sum_{n=1}^{n} I S_{m n j t} \leq \sum_{i=1}^{i} V C_{i m} \times P_{i j t} / 30 \times(1+\alpha) \times L T_{m}
$$

$\forall j, m, t$,

$$
\sum_{j=1}^{J} S_{m n t j} \leq C_{m n} \quad \forall m, n, t
$$$$
\sum_{j=1}^{J} S_{m n j t} \geq \beta \times \sum_{n=1}^{n} \sum_{j=1}^{J} S_{m n j t} \quad \forall m, n, t,
$$

$S_{m n j t}, I S_{m n j t} \geq 0$, integer $, \quad \forall m, n, j, t$.

\subsection{Robust-fuzzy counterpart model}

In this section, we present the final model by considering the uncertainty of decision makers in determining $\tilde{i}_{\sim}$ terval half-length of parameter $c_{i}$ as a fuzzy number, $\tilde{\hat{c}}_{i}$. Thus, by considering the innovative robust-fuzzy approach presented in Section 2.2, the nominal goal model is converted into the following robust-fuzzy counterpart model:

$\operatorname{Max} Z=\lambda$,

S.t. :

$$
\begin{aligned}
& \frac{w_{1} d_{1}^{+}}{G 1}+\frac{w_{2} d_{2}^{+}}{G 2}+\frac{w_{3} d_{3}^{-}}{G 3}+\left(Z^{1}-Z^{0}\right) \lambda \leq Z^{1}, \\
& \sum_{m=1}^{M} \sum_{n=1}^{N} \sum_{j=1}^{J} \sum_{t=1}^{T} C S_{m n} S_{m n t j}
\end{aligned}
$$$$
+\sum_{m=1}^{M} \sum_{n=1}^{N} \sum_{j=1}^{J} \sum_{t=1}^{T} \bar{C} t_{m n j} S_{m n t j}
$$$$
+\sum_{m=1}^{M} \sum_{n=1}^{N} \sum_{j=1}^{J} \sum_{t=1}^{T} C h_{m n} I S_{m n t j}
$$$$
+\sum_{m=1}^{M} \sum_{n=1}^{N} \sum_{j=1}^{J} \sum_{t=1}^{T} P P_{m n j t}
$$$$
+Z \times \Gamma_{1}-d_{1}^{+} \leq G_{1},
$$

$\sum_{i=1}^{I} \sum_{m=1}^{M} \sum_{n=1}^{N} \sum_{t=1}^{T} P P M_{m n} S_{m n t}+d_{2}^{-}-d_{2}^{+}=G_{2}$,

$\sum_{i=1}^{I} \sum_{m=1}^{M} \sum_{n=1}^{N} \sum_{t=1}^{T} D P_{m n} S_{m n t}+d_{3}^{-}-d_{3}^{+}=G_{3}$,

$$
\sum_{n=1}^{n} S_{m n j t}=\sum_{i=1}^{i} V C_{i m} P_{i j t}-\sum_{n=1}^{n} I S_{m n j t-1}
$$$$
+\sum_{n=1}^{i} I S_{m n j t} \quad \forall j, m, t
$$

$\sum_{n=1}^{n} I S_{m n j t} \geq \sum_{i=1}^{i} V C_{i m} \times P_{i j t} / 30 \times \alpha \times L T_{m}$

$\forall j, m, t$,

$\sum_{n=1}^{n} I S_{m n j t} \leq \sum_{i=1}^{i} V C_{i m} \times P_{i j t} / 30 \times(1+\alpha) \times L T_{m}$

$\forall j, m, t$, 


$$
\begin{aligned}
& \sum_{j=1}^{J} S_{m n j t}+Z Z_{m n t} \Gamma_{2}+p p p_{m n t} \leq \bar{C}_{m n} \quad \forall m, n, t \\
& \sum_{j=1}^{J} S_{m n j t} \geq \beta \times \sum_{n=1}^{n} \sum_{j=1}^{J} S_{m n j t} \quad \forall m, n, t, \\
& P P_{m n j t}+Z Z \geq \hat{C} t S_{m n j t}, \quad \forall m, n, j, t, \\
& Z Z_{m n t}+p p p_{m n t}+\lambda\left(C_{m e d}-C_{\min }\right) \geq C_{\min }, \quad \forall m, n, t \\
& S_{m n j t}, I S_{m n j t} \geq 0, \text { integer }, \quad \forall m, n, j, t .
\end{aligned}
$$

\section{Model parameters and data collection}

Model parameters (data) can generally be divided into two general categories:

- Certain parameters: production level of each product, purchase cost (price), inventory cost, the number of on-time deliveries, values of the goal, and goal's importance coefficients;

- Uncertain parameters: capacity and transportation cost.

By adopting ABC method and removing singlesource parts from the list, 129 suppliers were examined. As mentioned before, the model was implemented for only two vehicle models in manufacturing plant (Tehran). The robust model was solved based on threeperiodic (monthly) production plan.

In order to determine the priority of goals (objectives), we applied AHP technique. To do that, we collected the opinions of 10 top managers and experts of the company through AHP questionnaire, and then analyzed them by using Excel spreadsheets and Expert Choice software (see Table 1).

The inconsistency ratio was set equal to 0.04, although 0.1 was usually reliable for this index.

\section{Computational results}

After solving the model, the following three solutions, called Normal, Pessimistic, and Optimistic, were obtained.

- Normal solution: solving robust-fuzzy model in normal mode, namely, considering interval halflength of supplier capacity parameter in fuzzy form;

- Pessimistic solution: solving robust-fuzzy model with $\lambda=1$, namely, considering maximum amount

Table 1. Importance coefficients of goals.

\begin{tabular}{lccc}
\hline Objectives (goals) & First & Second & Third \\
Importance coefficient of goal & 0.364 & 0.327 & 0.309 \\
\hline
\end{tabular}

for interval half-length of supplier capacity parameter;

- Optimistic solution: solving robust-fuzzy model with $\lambda=0$, namely, considering minimum amount for interval half-length of supplier capacity parameter.

Pessimistic and optimistic solutions could show the accuracy of robust-fuzzy model's results. Obviously, Normal solution should be between Pessimistic and Optimistic solutions.

We considered $\Gamma_{1}$ and $\Gamma_{2}$ for 11 different values. Therefore, for each type of solution (Normal, Pessimistic, and Optimistic), 11 models were solved.

The role of protection level parameter, $\Gamma_{i}$, is usually set by model builder, according to risk preference of decision maker. Its value is equal the number of uncertain parameters of which the uncertainties should be considered by the model. In other words, value of parameter, $\Gamma_{i}$, is equal to the number of uncertain parameters that are allowed to violate their nominal values. For example, $\Gamma_{i}=0$ corresponds to an absolute conservative manager. For more information, refer to Bertsimas and Sim [33].

\subsection{Verifying the model by using Monte Carlo simulation}

To measure performance accuracy of the model (quality of robust solutions), and to calculate the risk, we ran a simulation model by randomly generating uncertain parameters 10000 times with different deterministic parameters. In general, a total of 33 simulation runs were performed in order to determine the number of constraints that were violated. In other words, for each protection level, the ratio of the total number of violated constraints to total constraints was determined.

The simulation model was implemented to test and validate the result obtained via the robust model. The outputs of simulation model are the probability of constraints violation or risk rate. For this research, the role of simulation and its relationship with mathematical model are shown in this form.

In fact, managers could determine the values of decision variables after selecting a desired risk rate.

\subsection{Results and analysis}

From Table 2 and Figure 6, it can be inferred that increasing the protection level causes the level of constraints satisfaction $(\lambda)$ to drop.

Figure 7 shows that the robust-fuzzy model values (Normal solution) are in balance compared with two other solutions (although all the three begin from the same point). On the other hand, it can be inferred that when the protection level increases, graphs (Figure 7) are further away from each other. Therefore, in these points, it is clear that interval half-lengths are fuzzy.

Table 2 and Figure 7 show that the levels of risk 
Table 2. The objective function values for Normal, Pessimistic, and Optimistic solutions.

\begin{tabular}{ccccccc}
\hline $\begin{array}{c}\text { Protection } \\
\text { levels }\end{array}$ & $\boldsymbol{\Gamma}_{\mathbf{1}}$ & $\boldsymbol{\Gamma}_{\mathbf{2}}$ & $\boldsymbol{\lambda}$ & $\begin{array}{c}\text { Sum of deviations } \\
\text { from goals }\end{array}$ & $\begin{array}{c}\text { Sum of deviations } \\
\text { from goals }\end{array}$ & $\begin{array}{c}\text { Sum of deviations } \\
\text { from goals }\end{array}$ \\
\hline 1 & 0 & 0.0 & 1 & 0.18335 & 0.18335 & 0.18335 \\
2 & 39 & 0.1 & 0.905 & 0.223651 & 0.225652 & 0.204661 \\
3 & 78 & 0.2 & 0.819 & 0.260208 & 0.267845 & 0.225725 \\
4 & 117 & 0.3 & 0.740 & 0.293585 & 0.310022 & 0.24683 \\
5 & 156 & 0.4 & 0.668 & 0.324197 & 0.352209 & 0.267928 \\
6 & 195 & 0.5 & 0.601 & 0.352368 & 0.39446 & 0.289015 \\
7 & 234 & 0.6 & 0.540 & 0.378407 & 0.436768 & 0.310102 \\
8 & 273 & 0.7 & 0.477 & 0.404853 & 0.479143 & 0.331194 \\
9 & 312 & 0.8 & 0.413 & 0.432246 & 0.521548 & 0.352286 \\
10 & 351 & 0.9 & 0.349 & 0.459137 & 0.563964 & 0.373407 \\
11 & 387 & 1.0 & 0.287 & 0.485414 & 0.607242 & 0.394537 \\
\hline
\end{tabular}

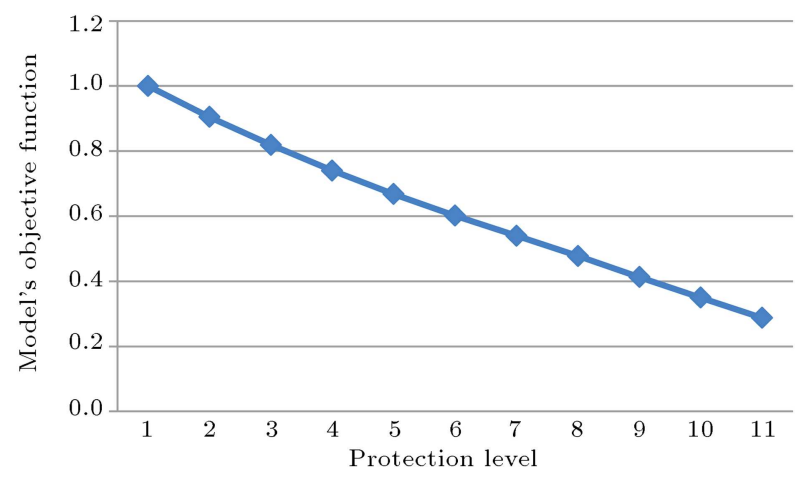

Figure 6. Behavior of objective function with respect to $(\lambda)$ in Normal solution.

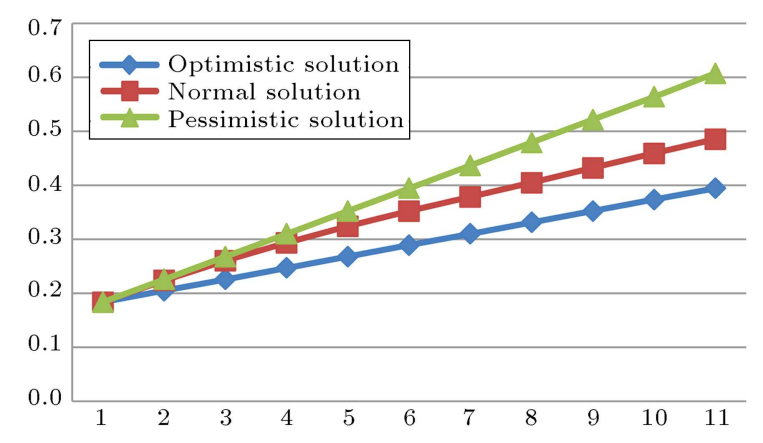

Figure 7. Behavior of sum of deviations from goals.

or conservation level highly affect the slopes of the lines of objective function values. Thus, the robust model, compared with the deterministic model, has more significant effects on risk reduction.

In Table 3, columns a-c show the percentages of deviation from goal 1 to 3 for 3 types of solutions, respectively.

In Table 4, columns a-c show the probability of

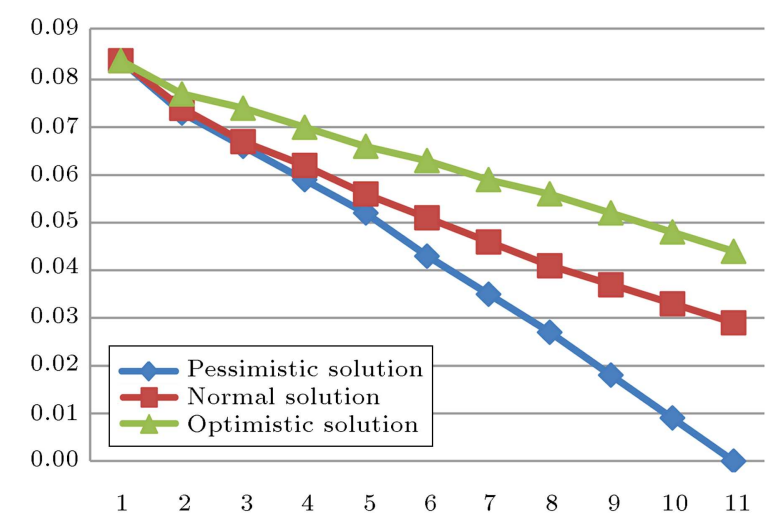

Figure 8. The trend of the probability of constraints violation in different modes based on Indicator 1.

first goal constraints violation, probability of capacity constraints violation, and the sum of probabilities of violation, respectively. Table 4 indicates the risk rate (probability of constraints violation) based on indicator 1.

From Table 2, it can be seen that increasing the protection levels decreases the objective function $(\lambda)$ and the probability of violation. In fact, as the level of protection increases, the model variable values will be chosen more stringently in the allowed interval so that probability of limitation violation and value of the objective function decrease. This could be a reason why the performance of robust modeling is accurate. The numbers in Table 4 are the outcome of simulation and show that increasing protection level decreases the probability of violation. This process indicates the accuracy of the performance and simulation of the robust model (see Figures 8 and 9).

The first goal constraint has 387 uncertain parameters and includes protection level of $\Gamma_{1}$. The other 
Table 3. The percentage of deviation from each goal.

\begin{tabular}{|c|c|c|c|c|c|c|c|c|c|c|c|}
\hline \multirow[b]{2}{*}{$\begin{array}{c}\text { Protection } \\
\text { levels }\end{array}$} & \multirow[b]{2}{*}{$\Gamma_{1}$} & \multirow[b]{2}{*}{$\Gamma_{2}$} & \multicolumn{3}{|c|}{ Normal solution } & \multicolumn{3}{|c|}{ Pessimistic solution } & \multicolumn{3}{|c|}{ Optimistic solution } \\
\hline & & & $\mathbf{a}$ & $\mathbf{b}$ & c & $\mathbf{a}$ & b & c & $\mathbf{a}$ & $\mathbf{b}$ & c \\
\hline 1 & 0 & 0.0 & 27.90 & 20.05 & 5.24 & 27.90 & 20.05 & 5.24 & 27.90 & 20.05 & 5.24 \\
\hline 2 & 39 & 0.1 & 28.00 & 20.11 & 18.11 & 28.00 & 20.11 & 18.76 & 28.00 & 20.08 & 12.00 \\
\hline 3 & 78 & 0.2 & 28.02 & 20.18 & 29.85 & 28.02 & 20.19 & 32.31 & 28.02 & 20.11 & 18.76 \\
\hline 4 & 117 & 0.3 & 28.03 & 20.24 & 40.57 & 28.03 & 20.27 & 45.86 & 28.03 & 20.15 & 25.54 \\
\hline 5 & 156 & 0.4 & 28.04 & 20.30 & 50.41 & 28.04 & 20.35 & 59.42 & 28.04 & 20.19 & 32.31 \\
\hline 6 & 195 & 0.5 & 28.04 & 20.35 & 59.46 & 28.03 & 20.45 & 73.00 & 28.04 & 20.23 & 39.09 \\
\hline 7 & 234 & 0.6 & 28.04 & 20.41 & 67.84 & 28.00 & 20.56 & 86.61 & 28.04 & 20.27 & 45.87 \\
\hline 8 & 273 & 0.7 & 28.03 & 22.32 & 74.38 & 27.98 & 20.63 & 100.26 & 28.04 & 20.31 & 52.65 \\
\hline 9 & 312 & 0.8 & 28.01 & 26.21 & 79.15 & 27.98 & 20.68 & 113.95 & 28.05 & 20.35 & 59.43 \\
\hline 10 & 351 & 0.9 & 29.34 & 28.36 & 84.01 & 27.97 & 20.73 & 127.63 & 28.04 & 20.40 & 66.22 \\
\hline 11 & 387 & 1.0 & 32.32 & 28.37 & 89.00 & 27.96 & 20.76 & 141.62 & 28.03 & 20.45 & 73.02 \\
\hline
\end{tabular}

Table 4. Probability of constraints violation (risk rates) in different modes based on Indicator 1.

\begin{tabular}{|c|c|c|c|c|c|c|c|c|c|c|c|}
\hline \multirow[b]{2}{*}{$\begin{array}{c}\text { Protection } \\
\text { levels }\end{array}$} & \multirow[b]{2}{*}{$\Gamma_{1}$} & \multirow[b]{2}{*}{$\Gamma_{2}$} & \multicolumn{3}{|c|}{ Normal solution } & \multicolumn{3}{|c|}{ Pessimistic solution } & \multicolumn{3}{|c|}{ Optimistic solution } \\
\hline & & & a & $\mathbf{b}$ & c & $\mathbf{a}$ & $\mathbf{b}$ & $\mathbf{c}$ & $\mathbf{a}$ & $\mathbf{b}$ & c \\
\hline 1 & 0 & 0.0 & 0.002 & 0.082 & 0.084 & 0.002 & .082 & 0.084 & 0.002 & 0.082 & 0.084 \\
\hline 2 & 39 & 0.1 & 0 & 0.074 & 0.074 & 0 & 0.073 & 0.073 & 0 & 0.077 & 0.077 \\
\hline 3 & 78 & 0.2 & 0 & 0.067 & 0.067 & 0 & 0.066 & 0.066 & 0 & 0.074 & 0.074 \\
\hline 4 & 117 & 0.3 & 0 & 0.062 & 0.062 & 0 & 0.059 & 0.059 & 0 & 0.07 & 0.070 \\
\hline 5 & 156 & 0.4 & 0 & 0.056 & 0.056 & 0 & 0.052 & 0.052 & 0 & 0.066 & 0.066 \\
\hline 6 & 195 & 0.5 & 0 & 0.051 & 0.051 & 0 & 0.043 & 0.043 & 0 & 0.063 & 0.063 \\
\hline 7 & 234 & 0.6 & 0 & 0.046 & 0.046 & 0 & 0.035 & 0.035 & 0 & 0.059 & 0.059 \\
\hline 8 & 273 & 0.7 & 0 & 0.041 & 0.041 & 0 & 0.027 & 0.027 & 0 & 0.056 & 0.056 \\
\hline 9 & 312 & 0.8 & 0 & 0.037 & 0.037 & 0 & 0.018 & 0.018 & 0 & 0.052 & 0.052 \\
\hline 10 & 351 & 0.9 & 0 & 0.033 & 0.033 & 0 & 0.009 & 0.009 & 0 & 0.048 & 0.048 \\
\hline 11 & 387 & 1.0 & 0 & 0.029 & 0.029 & 0 & 0 & 0 & 0 & 0.044 & 0.044 \\
\hline
\end{tabular}

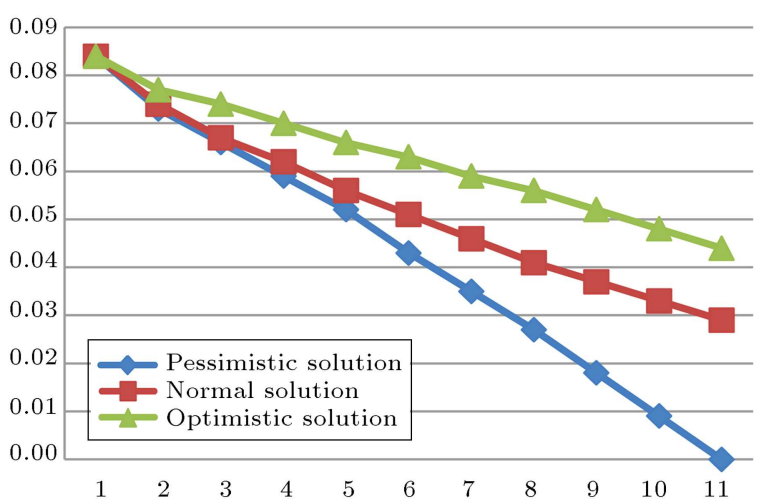

Figure 9. The trend of the probability of constraints violation in different modes based on Indicator 2 .

constraints, including uncertain parameters, consist of 387 capacity constraints with protection level of $\Gamma_{2}$.

Simulation results indicate that only some of the capacity constraints (up to a maximum of 73) are open to violation. The reason is that the capacity of some suppliers is more than needed or the model has considered a share which is less than the existing capacity so that the considered uncertainty for some parameters of the supplier cannot affect them. According to this explanation, two indicators are considered for risk calculation.

- Indicator 1. To divide the total number of violated states by the total number of states;

- Indicator 2. To divide the total number of violated states by the total number of possible states which are dependent on the maximum number of violable states.

Figures 10 and 11 demonstrate the trade-off between sum of deviations and probability of constraints violation based on indicator values 1 and 2 , respectively.

\section{Conclusion}

In this study, an innovative robust-fuzzy methodology was developed and applied to a real case study of supplier selection problem within automobile industry 


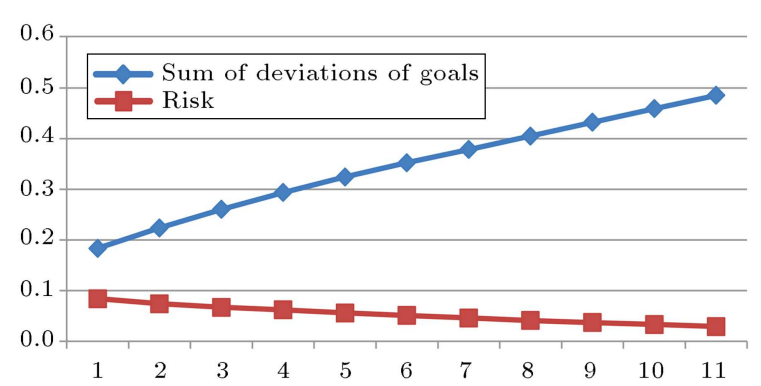

Figure 10. Trade-off between sum of deviations and probability of constraints violation based on Indicator 1.

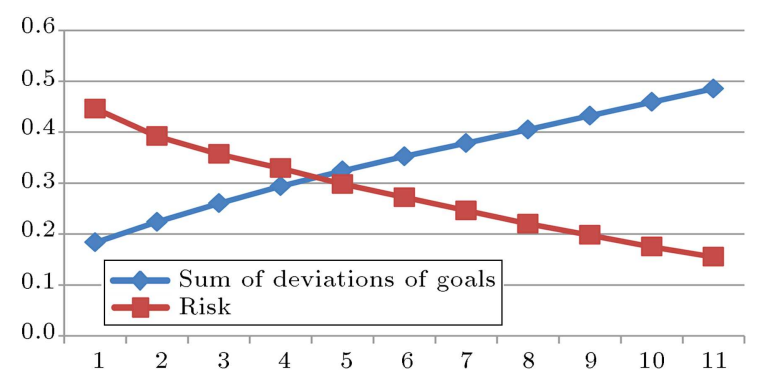

Figure 11. Trade-off between sum of deviations and probability of constraints violation based on Indicator 2 .

with multiple uncertainties. This approach was based on the integration of techniques of robust optimization, developed by Bertsimas and Sims' approach (2004), and fuzzy programming, based on Werners' approach (1978b), to handle two modes of uncertainty. In robust optimization models, uncertainty of parameters is modeled as random variables that take value within a symmetrical interval. In fact, our innovative robustfuzzy method was an improved version of robust optimization model. This approach was properly responsive to uncertainty of decision maker about interval half-length and could provide the necessary flexibility for the decision maker. In other words, this method was able to be responsive to both types (modes) of uncertainty.

The performance of the results of the proposed model in comparison with the results obtained via simulation techniques was proved to be reliable under various conditions. In other words, the proposed model could properly manage a variety of uncertain conditions and provide useful and reliable information for flexible decision making processes. The flexibility of the decision making process is shown in Figures 7-9.

Although this study is the first attempt to supplier selection decision making problem through this approach, the results suggest that it can also be extended from both theoretical and practical points of view.

In addition, the following managerial implications are of great importance: As stated in Sections 1, 2, and 3 , the issue of uncertainty is very important in supplier selection problem because of the inherent uncertainty associated with various factors such as capacity and transportation cost. Due to the high volume of required parts in this case study and considering multiple criteria as well as the condition of uncertainty, choosing suitable suppliers is of great importance.

In general, robust optimization concept and especially innovative robust-fuzzy method are easy to understand and implement, especially by automotive industry managers. Robust optimization approach is especially more practical and applicable in comparison with stochastic programming models. As explained before, in robust optimization approach, uncertain data take value within an interval. In some real cases, such as our case study, it is difficult for managers to determine the exact interval length. Furthermore, in these situations, the decision maker suggests an ambiguous interval half-length. To overcome this problem, we developed an innovative approach and proposed the interval half-length in terms of fuzzy number. The new method could help managers to achieve flexibility in decision making in case of ambiguous interval halflength. Consequently, the innovative method could be of great interest to managers as well as academics.

In addition, the following implications can be inferred from the mathematical model and computational results:

1. The mathematical model considered multiple criteria in the form of three objectives (goals). This could better match the real world. This model helps automotive industry supply chain managers to make integrated and comprehensive decisions considering various issues such as multiple products, multiple plants, multiple suppliers, and multiple decision-making periods;

2. Computational results demonstrated that managers with a desired risk level could make a suitable decision. In other words, as Tables 2, 3, and 4 indicate, they have 11 options to select. An optimal combination of suppliers can exist for each selection option;

3. The implementation of the proposed method did not require extensive and complex historical data; only determining an interval for uncertain parameters by experts was enough;

4. In this context, the improved decision making is related to robustness of solutions (see Figure 1). In uncertain environment, a proper decision is equivalent to a robust decision. In the proposed approach, the main decision is the value of supply rate of part $m$ from supplier $n$ in period $t$ for plant $j$ (value of $S_{m n j i}$ ). A robust value for this variable is equivalent to robust decision. Managers can determine this value after selecting a desired risk rate; 
5. Due to the nature of auto industry, the logistic manger has to manage thousands of parts and components. Obviously, the lack of even one part can stop the production line and impose an incredible cost as well as some intangible costs, such as customer dissatisfaction. From this point of view, this industry is much more sensitive to appropriate supplier selection than others. On the other hand, inherent uncertainty of supplier relationship is a crucial factor, especially in the developing countries. Therefore, this industry needs practical, efficient, and reliable methods to manage the logistics of parts and components.

The contribution of this research from the viewpoint of the value added to companies is presented as follows.

The innovative model is applicable and efficient for manufacturing companies in which the issue of uncertainty is highly significant. In other words, due to the existence of many important sources of uncertainties in business environment, reliable data cannot be found and the classical supply selection models are not applicable any more. Thus, our proposed method is applicable for the companies with uncertain input data. Although our study was planned for a large auto industry, it could be implemented in other industries such as financial, banking, insurance, etc.

The major limitation of the research is how to determine the membership function of fuzzy numbers. Furthermore, the model is not quite applicable to "make to order" manufacturing systems. Future research can extend this approach to cover other systems. Another issue in future research can be using artificial intelligence techniques instead of robust optimization methods.

\section{References}

1. Li, L. and Zabinsky, Z.B. "Incorporating uncertainty in to a supplier selection problem", Int. J. Prod. Econ., 134(2), pp. 344-356 (2011).

2. Nam, S.-H., Vitton, J., and Kurata, H. "Robust supply base management: Determining the optimal number of suppliers utilized by contractors", Int. J. Prod. Econ., 134(2), pp. 333-343 (2011).

3. Hasani, A., Zegordi, S.H., and Nikbakhsh, E. "Robust closed-loop supply chain network design for perishable goods in agile manufacturing under uncertainty", Int. J. Prod. Res., 50(16), pp. 1-21 (2011).

4. Liu, Y. and Peng, B. "Achieving robustness objectives within a supply chain by means of reliability allocation", In Proceedings of the 2009 International Symposium on Web Information Systems and Applications (WISA '09) Nanchang, P.R. China, pp. 210-213 (2009).

5. Amid, A., Ghodsypour, S.H., and O'Brien, C. "Fuzzy multiobjective linear model for supplier selection in a supply chain", Int. J. Prod. Econ., 104(2), pp. 394-407 (2006).

6. Benton, W.C. "Quantity discount decision under conditions of multiple items, multiple suppliers and resource limitation", Int. J. Prod. Econ., 27(1991), pp. 1953-1961 (2006).

7. Ghodsypour, S.H. and O'Brien, C. "A decision support system for reducing the number of suppliers and managing the supplier partnership in A JIT/TQM environment", The Proceeding of $3 r d$ International Symposium on Logistics, University of Padua, Italy (1997).

8. Ghodsypour, S.H. and O'Brien, C. "A decision support system for supplier selection using an integrated analytic hierarchy process and linear programming", Int. J. Prod. Econ., 56-57(1-3), pp. 199-212 (1998).

9. Ghodsypour, S.H. and O'Brien, C. "The total cost of logistics in supplier selection, under conditions of multiple sourcing, multiple criteria and capacity constraint", Int. J. Prod. Econ., 73(1), pp. 15-27 (2001).

10. Basnet, C. and Leung, J.M.Y. "Inventory lot-sizing with supplier selection", Comput. Oper. Res., 32(1), pp. 1-14 (2005).

11. Ustun, O. and AktarDemirtas, E. "An integrated multi-objective decision-making process for multiperiod lot-sizing with supplier selection", Omega, 36, pp. 509-521 (2008).

12. Mendoza, A. and Ventura, J.A. "Analytical models for supplier selection and order quantity allocation", Appl. Math. Model., 36, pp. 3826-3835 (2012).

13. Kumar, M., Vrat, P., and Shankar, R. "A fuzzy goal programming approach for vendor selection problem in a supply chain", Comput. Ind. Eng., 46(1), pp. 69-85 (2004).

14. Chen, C.-T., Lin, C.-T., and Huang, S.-F. "A fuzzy approach for supplier evaluation and selection in supply chain management", Int. J. Prod. Econ., 102(2), pp. 289-301 (2006).

15. Kilic, H.S. "An integrated approach for supplier selection in multi-item/multi-supplier environment", Appl. Math. Model., 37, pp. 7752-7763 (2013).

16. Nazari-Shirkohi, S., Shakouri, H., Javadi, B., and Keramati, A. "Supplier selection and order allocation problem using a two-phase fuzzy multi-objective linear programming", Appl. Math. Model., 37, pp. 9308-9323 (2013).

17. Roshandel, J., Miri-Nargesi, S.S., and HatamiShirkouhi, L. "Evaluating and selecting the supplier in detergent production industry using hierarchical fuzzy TOPSIS", Appl. Math. Model., 37, pp. 10170-10181 (2013).

18. Soufi Neyestani, M., Jolai, F., and Golmakani, H.R. "An evolutionary algorithm for supplier order allocation with fuzzy parameters considering linear and volume discount", Scientia Iranica E, 22(3), pp. 11301141 (2015). 
19. Mahmoudi, A., Sadi-Nezhad, and Makui, A. "An extended fuzzy VIKOR for group decision-making based on fuzzy distance to supplier selection", Scientia Iranica E, 23(4), pp. 1879-1892 (2016).

20. Kasilingam, R.G. and Lee, C.P. "Selection of vendorsa mixed-integer programming approach", Comput. Ind. Eng., 31, pp. 347-50 (1996).

21. Feng, C.-X., Wang, J., and Wang, J.S. "An optimization model for concurrent selection of tolerances and suppliers", Comput. Ind. Eng., 40(1-2), pp. 15-33 (2001).

22. Bonser, J.S. and Wu, S.D. "Procurement planning to maintain both short-term adaptiveness and long-term perspective", Manage. Sci., 47, pp. 769-786 (2001).

23. Seshadri, S., Chatterjee, K., and Lilien, G.L. "Multiple source procurement competitions", Market. Sci., 10, pp. 246-53 (1991).

24. Ranjbar Tezenji, F., Mohammadi, M., Pasandideh, S.H.R., and Nouri Koupaei, M. "An integrated model for supplier location-selection and order allocation under capacity constraints in an uncertain environment", Scientia Iranica E, 23(6), pp. 3009-3025 (2016).

25. Van Landeghem, H. and Vanmaele, H. "Robust planning: a new paradigm for demand chain planning", $J$. Oper. Manage., 20, pp. 769-783 (2002).

26. Bertsimas, D. and Sim, M. "Robust discrete optimization and network flows", Math. Programming Ser. B., 98, pp. 48-71 (2003).

27. Soyster, A.L. "Convex programming with set-inclusive constraints and applications to inexact linear programming”, Oper. Res., 21, pp. 1154-1157 (1973).

28. Ben-Tal, A. and Nemirovski, A. "Robust convex optimization", Math. Oper. Res., 23, pp. 769-805 (1998).

29. Ben-Tal, A. and Nemirovski, A. "Robust solutions of uncertain linear programs", Oper. Res. Lett., 25, pp. 1-13 (1999).

30. Ben-Tal, A. and Nemirovski, A. "Robust solutions of linear programming problems contaminated with uncertain data", Math. Programming, Ser. A., 88, pp. 411-424 (2000).
31. El-Ghaoui, L., Oustry, F., and Lebret, H. "Robust solutions to uncertain semidefinite programs", SIAM. J. Optim., 9, pp. 33-52 (1998).

32. El-Ghaoui, L. and Lebret, H. "Robust solutions to least-square problems to uncertain data matrices", SIAM. J. Matrix Anal. Appl., 18(4), pp. 1035-1064 (1997).

33. Bertsimas, D. and Sim, M., The Price of Robustness. Oper Rese, 52(1), pp. 35-53 (2004).

34. Werners, B. "Interactive multiple objective programming subject to flexible constraints", Euro. J. Oper Rese., 31, pp. 324-349 (1987).

35. Werners, B. "An interactive Fuzzy programming system", Fuzzy Sets and Systems., 23, pp. 131-147 (1987).

\section{Biographies}

Masood Rabieh received his $\mathrm{PhD}$ degree from Tarbiat Modaress University, Iran. He is an Assistant Professor in the Department of Industrial Management at Shahid Beheshti University, Iran. His research interests include operations research, system dynamics and system thinking, supply chain management, and project management.

Mohammad Modarres received his $\mathrm{PhD}$ degree from University of California, Los Angles (UCLA), USA. He is professor in the Department of Industrial Engineering at Sharif University of Technology, Iran. His research interests include operations research, mathematical modeling, and stochastic modeling.

Adel Azar received his $\mathrm{PhD}$ from University of Tehran, Iran. He is Professor in the Department of Industrial Management at Tarbiat Modaress University, Iran. His research interests include fuzzy management science, managerial and economic systems modeling, business process reengineering, system engineering, performance management, and performancebased budgeting. 